\title{
Oxaliplatin-based chemotherapy induces extravasated platelet aggregation in the liver
}

\author{
HIDEHIRO TAJIMA $^{1}$, TETSUO OHTA ${ }^{1}$, TOMOHARU MIYASHITA ${ }^{1}$, SHINICHI NAKANUMA $^{1}$, \\ MIKI MATOBA $^{1}$, TAKASHI MIYATA ${ }^{1}$, SEISHO SAKAI ${ }^{1}$, KOICHI OKAMOTO $^{1}$, ISAMU MAKINO ${ }^{1}$, \\ JUN KINOSHITA ${ }^{1}$, HIRONORI HAYASHI ${ }^{1}$, KEISHI NAKAMURA ${ }^{1}$, KATSUNOBU OYAMA ${ }^{1}$, \\ MASAFUMI INOKUCHI ${ }^{1}$, HISATOSHI NAKAGAWARA ${ }^{1}$, HIROYUKI TAKAMURA ${ }^{1}$, \\ HIROHISA KITAGAWA $^{1}$, SACHIO FUSHIDA ${ }^{1}$ and HIROKO IKEDA ${ }^{2}$
}

\author{
${ }^{1}$ Division of Cancer Medicine, Department of Gastroenterologic Surgery, Graduate School of Medical Science, \\ Kanazawa University; ${ }^{2}$ Department of Pathology, Kanazawa University Hospital, Kanazawa, Ishikawa 920-8641, Japan
}

Received October 24, 2014; Accepted January 30, 2015

DOI: $10.3892 / \mathrm{mco} .2015 .512$

\begin{abstract}
Oxaliplatin-based chemotherapy plays a central role in the treatment of patients with colorectal liver metastasis (CRLM). This treatment, however, has been associated with hepatic sinusoidal obstruction syndrome (SOS), a clinically important adverse effect characterized by a bluish hue of the liver, splenomegaly and thrombocytopenia, resulting in liver dysfunction. The significant association between the sinusoidal endothelium and platelets has suggested that oxaliplatin-based chemotherapy affects platelets in the liver. This study compared platelet counts in patients who did and did not receive oxaliplatin-based neoadjuvant chemotherapy (NAC). The peripheral blood platelet count was significantly lower in the NAC group $(n=17)$ compared to that in the non-NAC, or control group $(\mathrm{n}=15)(\mathrm{P}<0.05)$. The spleen index was also higher in the NAC group, although the difference was not significant. However, the spleens of the patients in the NAC group were significantly enlarged following treatment $(\mathrm{P}<0.01)$. Immunostaining for the platelet surface marker CD42b (glycoprotein Ib), revealed more platelets in the liver in the NAC compared to the control group, particularly in the centrilobular zone III, adjacent to the hepatic central vein and in contact with hepatocytes $(\mathrm{P}<0.01)$. The platelets present in the spaces of Disse, referred to as extravasated platelet aggregation (EPA), secrete a number of growth factors, including transforming growth factor- $\beta$, vascular endothelial growth factor-A, plasminogen activator inhibitor-1 and thromboxane A2. In conclusion, EPA may play an important role in the development of hepatic SOS. Moreover, antiplatelet drugs
\end{abstract}

Correspondence to: Dr Hidehiro Tajima, Division of Cancer Medicine, Department of Gastroenterologic Surgery, Graduate School of Medical Science, Kanazawa University, 13-1 Takaramachi street, Kanazawa, Ishikawa 920-8641, Japan E-mail: hidetaji@staff.kanazawa-u.ac.jp

Key words: colorectal liver metastasis, extravasated platelet aggregation, sinusoidal obstruction syndrome, oxaliplatin may prevent the onset of SOS and hepatic injury in patients treated with oxaliplatin-based chemotherapy for CRLM.

\section{Introduction}

Liver resection is the only treatment that offers hope of long-term survival and a cure for patients with colorectal liver metastasis (CRLM). Systemic neoadjuvant chemotherapy (NAC) may be used prior to conversion surgery and achieve $\mathrm{R} 0$ resection, even in patients with initially unresectable CRLM. Oxaliplatin-based chemotherapy plays a central role in the treatment of CRLM in Japan. However, oxaliplatin-based chemotherapy has been associated with sinusoidal obstruction syndrome (SOS), a condition characterized by hepatic sinusoidal dilatation, hepatocyte atrophy, perisinusoidal fibrosis and nodular regenerative hyperplasia (1). These histological changes have been observed in $\leq 40 \%$ of patients treated with oxaliplatin-based regimens undergoing liver resection (2-6). SOS is a cause for concern prior to major hepatectomy, as it is associated with increased perioperative morbidity and prolonged hospital stay (7). Clinically, the diagnostic factors associated with SOS include portal hypertension, splenomegaly, thrombocytopenia, abnormal indocyanine green (ICG) retention rate and elevations in the levels of liver enzymes, bilirubin and hyaluronic acid (8-12). Pathologically, SOS is characterized by disruption of the sinusoidal endothelium, collagen deposition in the perisinusoidal space, fibrosis, particularly around the central vein (in zone III), with dilatation of the sinusoidal space and congestion (13). The significant association between the sinusoidal endothelium and platelets (14) suggests platelet involvement in SOS and that oxaliplatin-based chemotherapy affects platelets in the liver. The aim of the present study was to assess the effects of oxaliplatin-based NAC on platelets in the liver.

\section{Material and methods}

Patients. Between January, 2005 and December, 2010, 32 patients with CRLM underwent surgery at the Department of Gastroenterologic Surgery, Kanazawa University Hospital 
(Kanazawa, Japan), including 17 patients who received oxaliplatin-based NAC (NAC group) and 15 who did not (control group). The patient records were retrospectively assessed and the factors evaluated included platelet count and indocyanine green (ICG) retention rate at $15 \mathrm{~min}$. Spleen volume was determined from computed tomography scans using a spleen index (15), which was calculated as 0.8 times the product of the long and short diameters of the maximum cut surface of the spleen.

Written informed consent was obtained from all the patients prior to their enrollment in the study. Treatment protocols were approved by the local Medical Ethics Committee.

Pathological specimens. Formalin-fixed, paraffin-embedded specimens were retrieved from the surgical pathology files of the Pathology Department of Kanazawa University Hospital.

Immunohistochemical examination. Immunohistochemical staining was performed using the Dako EnVision system, which uses dextran polymers conjugated with horseradish peroxidase (Dako, Carpinteria, CA, USA), thus avoiding any contamination by endogenous biotin. The tissues were fixed in $10 \%$ formaldehyde in phosphate-buffered saline, embedded in paraffin and cut into 5-mm tissue sections. The sections were deparaffinized in xylene and rehydrated in a graded ethanol series. Endogenous peroxidases were blocked by immersing the sections in $3 \% \mathrm{H}_{2} \mathrm{O}_{2}$ in $100 \%$ methanol for $20 \mathrm{~min}$ at room temperature. Antigen retrieval was achieved by microwaving the sections at $95^{\circ} \mathrm{C}$ for $10 \mathrm{~min}$ in $0.001 \mathrm{M}$ citrate buffer ( $\mathrm{pH}$ 6.7). After blocking endogenous peroxidases, the sections were incubated with Protein Block Serum-Free (Dako) at room temperature for $10 \mathrm{~min}$ to block non-specific staining. The sections were subsequently incubated for $2 \mathrm{~h}$ at room temperature with a 1:50 dilution of mouse monoclonal antibody against CD42b (cat. no. EPR6995; Abcam, Tokyo, Japan). Peroxidase activity was detected with the enzyme substrate 3-amino-9-ethylcarbazole. As negative controls, the sections were incubated with Tris-buffered saline without the primary antibody. Samples in which $\geq 10 \%$ of the tumor cells were slightly counterstained with Meyer's hematoxylin were defined as positive. Positive expression was defined as staining of $>5 \%$ of the cells.

Statistical analysis. Categorical variables were compared using the Chi-square test. All the tests were two-tailed and $\mathrm{P}<0.05$ was considered to indicate a statistically significant difference.

\section{Results}

Patient characteristics. Between January, 2005 and December, 2010, 32 patients with CRLM (25 men and 7 women) underwent surgery. Of those, 17 patients (13 men and 4 women) of mean age 64.3 years (range, 48-78 years), underwent oxaliplatin-based NAC (NAC group), whereas the remaining 15 patients (12 men and 3 women) of mean age 63.0 years (range, 37-83 years) underwent surgical resection alone (control group). The platelet levels were significantly lower in the NAC compared to those in the control group. The ICG retention rate differed between the two groups, although the difference was not statistically significant. The
Table I. Common biomarkers of sinusoidal obstruction syndrome.

\begin{tabular}{lccc}
\hline Markers & $\begin{array}{c}\text { NAC } \\
(\mathrm{n}=17)\end{array}$ & $\begin{array}{c}\text { Control } \\
(\mathrm{n}=15)\end{array}$ & P-value \\
\hline ICG 15 $(\%)$ & $10.9 \pm 6.7$ & $8.6 \pm 7.8$ & $\mathrm{NS}$ \\
Platelet count $\left({\left.\mathrm{x} 10^{4}\right)}^{2}\right.$ & $16.9 \pm 5.5$ & $22.7 \pm 8.8$ & $<0.05$ \\
Spleen index $\left(\mathrm{cm}^{2}\right)$ & $29.6 \pm 11.2$ & $27.2 \pm 10.4$ & $\mathrm{NS}$ \\
\hline
\end{tabular}

The values are presented as mean \pm standard deviation. NAC, neoadjuvant chemotherapy; ICG, indocyanine green; NS, not significant.

Table II. Spleen index of NAC cases $(n=17)$.

\begin{tabular}{lccc}
\hline Measurement & Prior to NAC & After NAC & P-value \\
\hline Spleen index $\left(\mathrm{cm}^{2}\right)$ & $25.6 \pm 10.4$ & $29.6 \pm 11.2$ & $<0.01$ \\
\hline
\end{tabular}

The values are presented as mean \pm standard deviation. NAC, neoadjuvant chemotherapy.

Table III. Immunohistochemical detection of CD42b in the resected liver.

\begin{tabular}{lccc}
\hline Zone & $\begin{array}{c}\text { NAC, no. }(\%) \\
(\mathrm{n}=15)\end{array}$ & $\begin{array}{c}\text { Control, no. }(\%) \\
(\mathrm{n}=12)\end{array}$ & P-value \\
\hline I & $4(26.7)$ & $1(8.3)$ & $\mathrm{NS}$ \\
II & $7(46.7)$ & $3(25.0)$ & $\mathrm{NS}$ \\
III & $9(60.0)$ & $1(8.3)$ & 0.009 \\
\hline
\end{tabular}

NAC, neoadjuvant chemotherapy; NS, not significant.

splenic index, a measure of spleen size, was higher in the NAC compared to that in the control group, but the difference was not significant (Table I). However, the spleens of the patients in the NAC group were significantly enlarged following oxaliplatin-based chemotherapy (Table II).

Immunohistochemical examination for CD42b. Tissue specimens surgically resected from 30 patients with CRLM (17 from the NAC and 13 from the control group), were immunohistochemically assayed for CD42b expression (Table III). The platelet counts were higher in the NAC compared to those in the control group, particularly in zone III $(\mathrm{P}=0.009)$. Moreover, platelet numbers were found to be correlated with the severity of SOS; specimens from patients with mild cases of SOS showed a few platelets in the sinusoidal space, whereas specimens from patients with severe SOS showed high platelet numbers in contact with hepatocytes, particularly in zone III (Fig. 1).

\section{Discussion}

SOS, also referred to as hepatic veno-occlusive disease, is a fatal hepatic injury that occurs predominantly following drug 

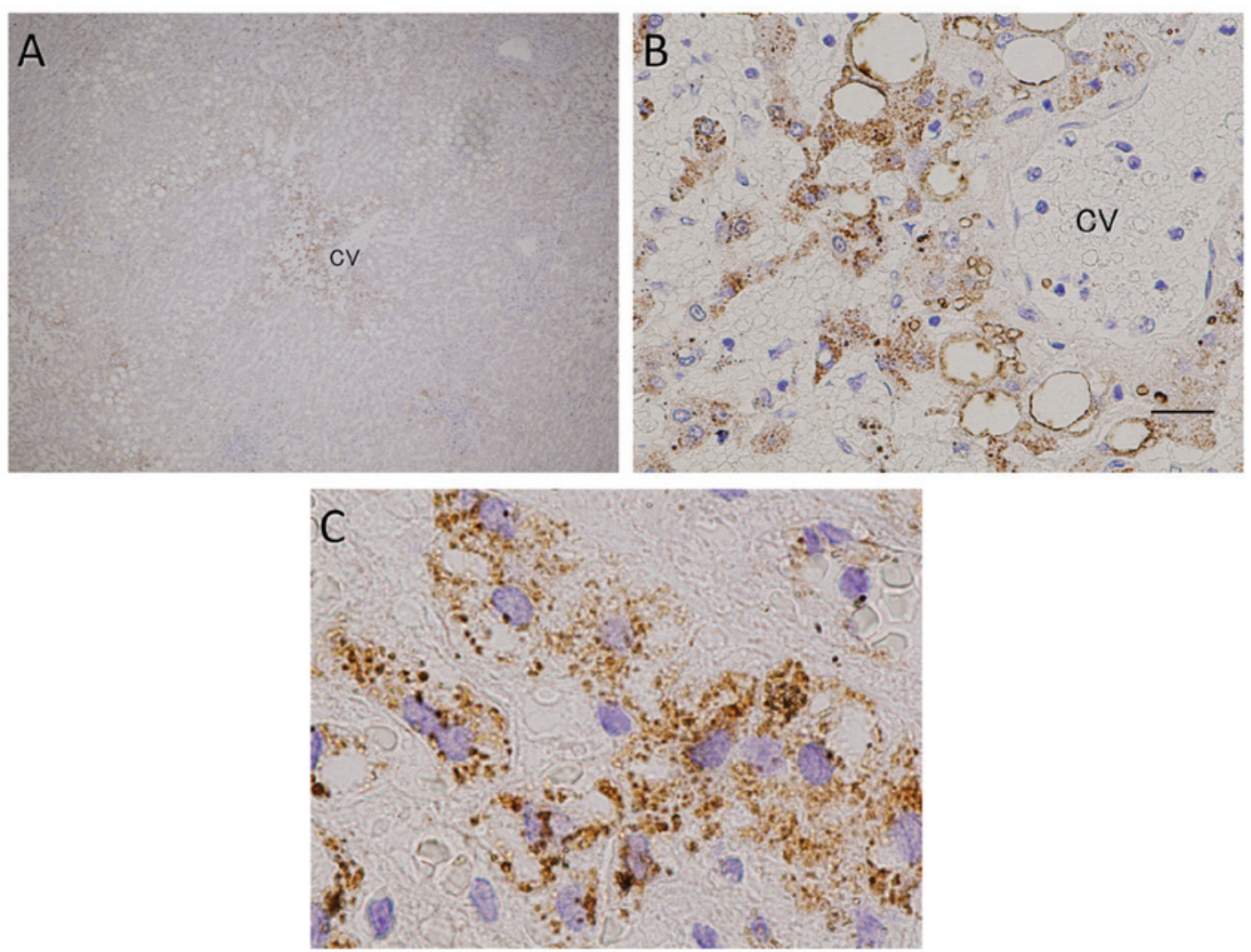

Figure 1. Immunohistochemical analysis of CD42b in hepatic tissue resected following preoperative chemotherapy with oxaliplatin. (A) CD42b staining was observed mainly around the central vein (CV), in zone III. (B) CD42b was detected in contact with hepatocytes, particularly in zone III; destruction of hepatocytes was also observed. Scale bar, $50 \mu \mathrm{m}$. (C) At higher magnification, CD42b was present as aggregates attached to hepatocytes along the sinusoid.

or toxin exposure. SOS may present in an acute, subacute or chronic form, usually with abdominal pain and hepatosplenomegaly, with evidence of portal hypertension, serum liver enzyme elevations and jaundice. Liver histology reveals sinusoidal obstruction in zone III, with hepatocyte necrosis and hemorrhage. Agents that cause SOS include cancer chemotherapeutic agents, particularly alkylating agents such as busulfan, cyclophosphamide and platinum coordination complexes - carboplatin, cisplatin and oxaliplatin. The diagnosis of SOS is usually based on its typical clinical presentation, which is characterized by hepatomegaly, ascites, portal hypertension, weight gain and jaundice (16), or exclusion of other causes of liver injury. In bone marrow transplant recipients, the usual differential diagnosis includes graft vs. host disease, sepsis, other forms of drug-induced liver injury and viral hepatitis. The diagnosis is usually supported by imaging, revealing changes typical of sinusoidal obstruction. Liver biopsy is diagnostic, but not usually necessary, and may be difficult due to concurrent coagulopathy or thrombocytopenia (13).

Oxaliplatin has been found to induce hepatic sinusoidal injury (HSI), defined as disruption of the sinusoidal endothelium and collagen deposition in the perisinusoidal space. HSI may result in portal hypertension, splenomegaly and, finally, thrombocytopenia. However, our finding of an early change in platelet levels suggests that thrombocytopenia occurs at an earlier stage.

Platelets are invisible on tissue samples stained with hematoxylin and eosin, as they have no nuclei. Immunostaining of the platelet surface marker CD42b (glycoprotein Ib $\alpha$ ), may be used to visualize the presence of platelets (17). Normal liver tissues exhibit maintenance of hepatocyte structure, the space of Disse and sinusoidal endothelium, with blood cells, including platelets, present only in the sinusoidal spaces. We observed that, in patients with mild SOS, a few platelets were present in the sinusoidal spaces and the hepatocytes remained intact. In patients with severe SOS, however, platelets were observed in contact with hepatocytes, particularly in zone III, with hepatocyte destruction.

These results suggested that the disruption of sinusoidal endothelial cells by oxaliplatin allows activated platelets to migrate into the space of Disse and aggregate. These activated platelets secrete various growth factors, including platelet-activating factor (PAF), thromboxane (TX)A2, thrombospondin, vascular endothelial growth factor (VEGF)-A and plasminogen activator inhibitor (PAI)-1 (18), which may cause the liver injury observed in SOS. PAF and TXA2 may cause central vein occlusion and portal hypertension (19). Transforming growth factor (TGF)- $\beta$, activated by thrombospondin (20), causes collagen deposition in the perisinusoidal space and inhibits substance exchange in the space of Disse, resulting in bilirubin elevation and an abnormal ICG retention rate. PAI-1 and TGF- $\beta$ interfere with liver regeneration by suppressing hepatocyte growth factor (21). VEGF-A, which usually acts as a vasodilator, may paradoxically act as a vasoconstrictor under conditions of endothelial failure (22). Moreover, bevacizumab, an antibody against VEGF-A, was found to protect against SOS in patients administered oxaliplatin-based chemotherapy for CRLM (23-25). 
In summary, treatment of CRLM patients with oxaliplatin-based NAC induces thrombocytopenia, along with platelet aggregation in zone III. This extravasated platelet aggregation in the space of Disse, particularly around the central vein, may play an important role in the development of SOS. Moreover, antiplatelet drugs may prevent the onset of SOS in patients administered oxaliplatin-based chemotherapy for CRLM.

\section{References}

1. Rubbia-Brandt L, Audard V, Sartoretti P, et al: Severe hepatic sinusoidal obstruction associated with oxaliplatin-based chemotherapy in patients with metastatic colorectal cancer. Ann Oncol 15: 460-466, 2004.

2. Hubert C, Fervaille C, Sempoux C, et al: Prevalence and clinical relevance of pathological hepatic changes occurring after neoadjuvant chemotherapy for colorectal liver metastases. Surgery 147: 185-194, 2010.

3. Vauthey JN, Pawlik TM, Ribero D, et al: Chemotherapy regimen predicts steatohepatitis and an increase in 90-day mortality after surgery for hepatic colorectal metastases. J Clin Oncol 24 2065-2072, 2006.

4. Tamandl D, Klinger M, Eipeldauer S, et al: Sinusoidal obstruction syndrome impairs long-term outcome of colorectal liver metastases treated with resection after neoadjuvant chemotherapy. Ann Surg Oncol 18: 421-430, 2011.

5. Robinson SM, Wilson CH, Burt AD, Manas DM and White SA Chemotherapy-associated liver injury in patients with colorectal liver metastases: a systematic review and meta-analysis. Ann Surg Oncol 19: 4287-4299, 2012.

6. Aloysius MM, Zaitoun AM, Beckingham IJ, et al: The pathological response to neoadjuvant chemotherapy with FOLFOX-4 for colorectal liver metastases: a comparative study. Virchows Arch 451: 943-948, 2007.

7. Nakano H, Oussoultzoglou E, Rosso E, et al: Sinusoidal injury increases morbidity after major hepatectomy in patients with colorectal liver metastases receiving preoperative chemotherapy. Ann Surg 247: 118-124, 2008.

8. Jardim DL, Rodrigues CA, Novis YAS, Rocha VG and Hoff PM: Oxaliplatin-related thrombocytopenia. Ann Oncol 23: 1937-1942, 2012.

9. Miura K, Nakano H, Sakurai J, et al: Splenomegaly in FOLFOX-naive stage IV or recurrent colorectal cancer patients due to chemotherapy-associated hepatotoxicity can be predicted by the aspartate aminotransferase to platelet ratio before chemotherapy. Int J Oncol 16: 257-263, 2011.

10. Krieger PM, Tamandl D, Herberger B, et al: Evaluation of chemotherapy-associated liver injury in patients with colorectal cancer liver metastases using indocyanine green clearance testing. Ann Surg Oncol 18: 1644-1650, 2011.

11. Sato S, Nakano H, Ishida $\mathrm{Y}$ and Otsubo T: The aspartate aminotransferase to platelet ratio before chemotherapy predicts adverse events for FOLFOX and XELOX regimens including bevacizumab as the first-line therapy for stage IV, recurrent and metastatic colorectal cancer. J Gastrointest Oncol 4: 203-209, 2013.
12. Narita M, Oussoultzoglou E, Chenard MP, et al: Liver injury due to chemotherapy-induced sinusoidal obstruction syndrome is associated with sinusoidal capillarization. Ann Surg Oncol 19: 2230-2237, 2012.

13. Morine Y, Shimada M and Utsunomiya T: Evaluation and management of hepatic injury induced by oxaliplatin-based chemotherapy in patients with hepatic resection for colorectal liver metastasis. Hepatol Res 44: 59-69, 2014.

14. Lalor PF, Herbert J, Bicknell R and Adams DH: Hepatic sinusoidal endothelium avidly binds platelets in an integrin-dependent manner, leading to platelet and endothelial activation and leukocyte recruitment. Am J Physiol Gastrointest Liver Physiol 304: G469-G478, 2013.

15. Koga T: Correlation between sectional area of the spleen by ultrasonic tomography and actual volume of the removed spleen. J Clin Ultrasound 7: 119-120, 1979.

16. Campos-Varela I, Castells L, Dopazo C, et al: Transjugular intrahepatic portosystemic shunt for the treatment of sinusoidal obstruction syndrome in a liver transplant recipient and review of the literature. Liver Transpl 18: 201-205, 2012.

17. Taaning E: Platelet immunology. ELISA for detection of platelet antibodies, platelet-specific antigens and platelet glycoproteins. Dan Med Bull 39: 343-354, 1992.

18. Battinelli EM, Markens BA and Italiano JE: Release of angiogenesis regulatory proteins from platelet alpha granules: modulation of physiologic and pathologic angiogenesis. Blood 118: 1359-1369, 2011.

19. Cui S, Shibamoto T, Liu W, Takano H and Kurata Y: Effects of platelet-activating factor, thromboxane A2 and leukotriene D4 on isolated perfused rat liver. Prostaglandins Other Lipid Mediat 80: 35-45, 2006.

20. Venkatraman L, Chia SM, Narmada BC, et al: Plasmin triggers a switch-like decrease in thrombospondin-dependent activation of TGF- $\beta 1$. Biophys J 103: 1060-1068, 2012.

21. Narmada BC, Chia SM, Tucker-Kellogg L and Yu H: HGF regulates the activation of TGF- $\beta 1$ in rat hepatocytes and hepatic stellate cells. J Cell Physiol 228: 393-401, 2013.

22. Parenti A, Brogelli L, Filippi S, Donnini S and Ledda F: Effect of hypoxia and endothelial loss on vascular smooth muscle cell responsiveness to VEGF-A: role of flt-1/VEGF-receptor-1. Cardiovasc Res 55: 201-212, 2002.

23. Ribero D, Wang H, Donadon M, et al: Bevacizumab improves pathologic response and protects against hepatic injury in patients treated with oxaliplatin-based chemotherapy for colorectal liver metastases. Cancer 110: 2761-2767, 2007.

24. Rubbia-Brandt L, Lauwers GY, Wang H, et al: Sinusoidal obstruction syndrome and nodular regenerative hyperplasia are frequent oxaliplatin-associated liver lesions and partially prevented by bevacizumab in patients with hepatic colorectal metastasis. Hepatology 56: 430-439, 2010.

25. van der Pool AEM, Marsman HA, Verheij J, et al: Effect of bevacizumab added preoperatively to oxaliplatin on liver injury and complications after resection of colorectal liver metastases. J Surg Oncol 106: 892-897, 2012. 